\title{
Real time monitoring of proportional myo-control by antagonist co-activation
}

\author{
Abdelali Laamarti ${ }^{1}$, Pr Abdelhakim Lakhdar ${ }^{2}$, Pr Omar Battas ${ }^{3}$, Pr Imane Kermadi ${ }^{4}$ and Pr Mohammed Abdoh Rafai ${ }^{5}$ \\ ${ }^{1}$ Laboratory research on nervous system, neurosensorial diseases and handicap. \\ Rue Tarik Ibnou Ziad, \\ Casablanca 20250, Maroc \\ ${ }^{2}$ Laboratory research on nervous system, neurosensorial diseases and handicap. \\ Rue Tarik Ibnou Ziad, \\ Casablanca 20250, Maroc \\ ${ }^{3}$ Laboratory research on Neurosciences and mental disease. \\ Rue Tarik Ibnou Ziad, \\ Casablanca 20250, Maroc \\ ${ }^{5}$ Laboratory research on nervous system, neurosensorial diseases and handicap. \\ Rue Tarik Ibnou Ziad, \\ Casablanca 20250, Maroc
}

\begin{abstract}
Surface electromyography (SEMG) signals from support muscles can be used for the myoelectrical control of the hand and forearm. However, in cases of neuropathy, SEMG signals are negligible (lower than background noise) or non-existent, which hampers myoelectric control of limbs.

In this paper, we prove that SEMG signals from the upper limb can support secondary information by antagonist coactivation for proportional myocontrol of the exoskeleton to help movement in cases of neuropathy in the forearm (e.g., CMT).
\end{abstract}

Keywords: exohand, exoskeleton myoelectric, real time myo control

\section{Introduction}

Non-invasive neuronal interface is important for people with reduced mobility, who can benefit from the help and support provided by myo-electric equipment. The myo-control of these devices is based on surface electromyography (SEMG) signals of peripheral nerves[1,2]. This type of control has been used since the 1970s [3] in myo-electric prosthetics of amputated limbs (hands, forearms, arms). Neuronal activity in the amputated limb is still detectable (called the phantom limb phenomenon), and through recognition and classification software, we can identify the desired movements and their characteristics (force angle, delay,..).

However, during peripheral nerve neuropathy, the direct use of SEMG from paralysed muscles is almost impossible, necessitating the development of solutions that involve neuro-motor intervention at the CNS level. This involves two methods for measuring neuronal activity:

1. Invasive: Using arrays of electrodes implanted in the brain.

2. Non-Invasive: Using EEG signals (Electroencephalogram)

Movement intention can be detected by classifying EEG signals using complicated software based on ANN (Artificial Neuronal Network) or fuzzy logic[3,4]. The preprocessing of the signal, the extraction of the characteristics, the classification, all these areas have evolved considerably. An overall view of this evolution is given in [5-7]. The introduction by Hudgins et al. [8] of a set of temporal domain (TD) functions in the pattern recognition systems allows wide adoption of this myoelectric control scheme. The addition of a simple 
linear classifier discriminant analysis [9], gave a reasonable reliability of detecting the intention of the user's motion [4,10-11]. On the other hand, the determination of the characteristics of the movement (speed, acceleration) were less evident. In fact the natural ability of the CNS to modulate electromyogram (EMG) signals is a practical means of controlling movement regardless of destination or speed and their temporal evolution. This method is generally called the real time proportional myo-control.

In practice, a simple weighted Mean Absolute Value (MAV) of the EMG signals that are normalized for each class of motion [12-14] allows a real-time proportional myo-electric control. Recent studies [15] have shown that myo-control performance by the pattern recognition system is superior to that of the on/off binary system in a position tracking test but was less than that of a conventional direct control system that used two electrode sites on pairs of antagonist muscles to determine direction and velocity.

This scenario precludes patients, who suffer from a motor deficit caused by neuropathy, from benefiting from the help and support of myo-electrical equipment. Thus, we wanted to identify a non-invasive neuronal interface, which allows the CNS to easily adapt to myo-controlled equipment. In other words, instead of attempting to formalise the relationship between the SEMG signal and intention, we took an alternate approach that allows the CNS to myo-control a system and improve this control via visual and sensory biofeedback. This method only requires the system to extract SEMG signal characteristics for a proportional myo-electric signal output. This means that the system has both the advantages of being non-invasive and inexpensive, which allows a large population of people with reduced mobility to recover a good part of their motor deficit.

In this article, we demonstrate that the CNS can transmit its motor intention of moving a muscle paralyzed by neuropathy via antagonist co-activation by extracting SEMG signals from peripheral nerves of muscles of a healthy joint, which are naturally involved in the motor action of the paralyzed joint. Indeed, visual and sensory biofeedback systems allow neuro-motor programming of the CNS to control motor planning of a paralysed joint (angle, force, speed, acceleration, etc.,) based on rapid extraction $(<10 \mathrm{~ms})$ of the antagonist co-activation signal. This results in an almost perfect counter reaction of the neuro-motor system for supporting reflexes $(>100 \mathrm{~ms})$ and protects joints from any dislocation (exceeding angle, speed, acceleration, or force).

\section{Methodology}

The CNS converts motor intention into motor planning of all the muscles required for the desired action. Motor planning of a muscle involves the temporal evolution of a few tens of innervating muscle fibres of several types, depending on the nature of the movement to be performed. The execution of this plan is controlled at four levels (see Fig 1):

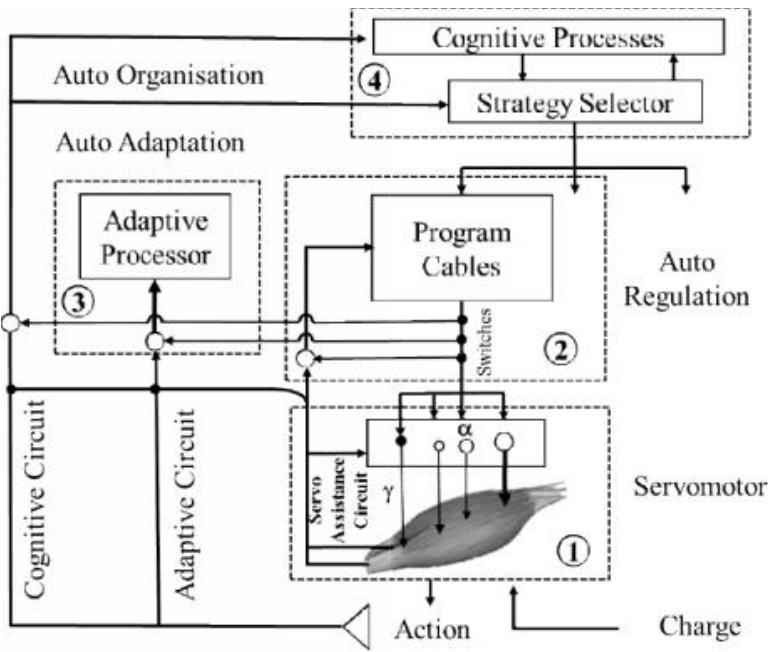

Fig 1: The hierarchy of control levels and their actions: servo assistance, regulation, adaptation, organisation (according to Paillard 1982a)

1. Servo assistance

2. Regulation

3. Adaptation

4. Organisation

These controls rely on visual-sensory biofeedback to adjust the controls for perfect execution without risking the outcome of desired movement.

Thus, in the case of sensorimotor disease or amputation, the lack of a servo-assistance circuit results in an unregulated servomotor, which causes deprogramming, rendering the detection of the motor intention unreliable. The most recent detection methods involve three essential steps (see Fig 2): 


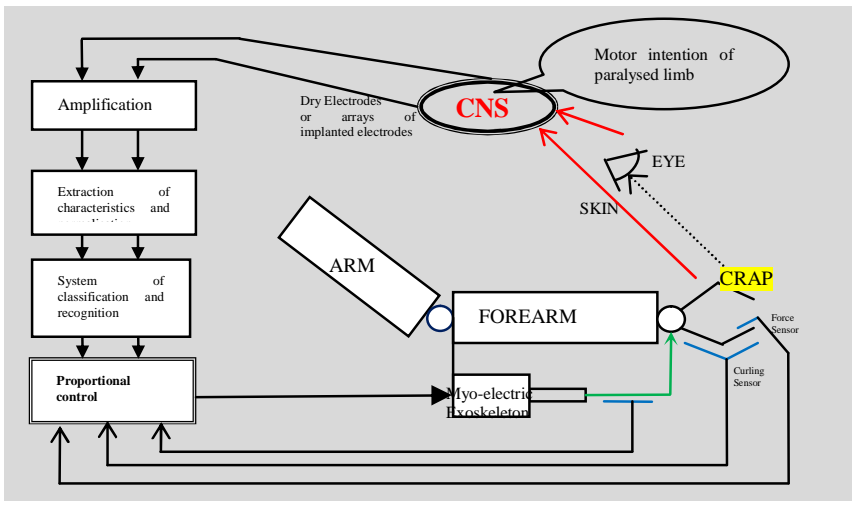

Fig 2: A forearm neuropathy case: the detection of motor intention for hand closing is based either on ECG signals or arrays of electrodes implanted in the brain. The normalised characteristics of these signals are fed into the artificial intelligence system for classification and recognition. This generates a proportional command for opening and closing of the hand. The exoskeleton is counteracted at the level of force and angle to protect the hand.

\subsection{Signal Measurement}

The location and method of measurement differs depending on the cause of the motor deficit. There are two important locations: peripheral nerves and the central nervous system, and two principal methods: invasive and non-invasive. The following table shows the methods used based on the location and type of measurement.

\begin{tabular}{|l|l|l|}
\hline & Invasive & Non invasive \\
\hline $\begin{array}{l}\text { Peripheral } \\
\text { Nerves }\end{array}$ & EMG needle & Surface EMG \\
\hline $\begin{array}{l}\text { Central Nervous } \\
\text { System }\end{array}$ & $\begin{array}{l}\text { Implant arrays of } \\
\text { electrodes }\end{array}$ & EEG \\
\hline
\end{tabular}

\subsection{Shape recognition}

Table 1: Margin specifications

Post amplification and digitization, the signal is sent to a processor to undergo two operations needed for shape recognition: the extraction of characteristics followed by classification based on artificial intelligence software and fuzzy logic (e.g., ANN). This classification allows detection of the motor intention of the patient.

\subsection{Generation of commands}

Based on the quality of the detection of intention, a number of TOR commands (all or none) or proportional commands (0 to $100 \%$ ) are generated.

The above methods have two major disadvantages:
1. The time for generating the servomotor command depends on the duration of the three treatment steps and the number of channels to measure the signals. Despite the availability of a new generation of processors, the treatment time is always greater than $200 \mathrm{~ms}$. This delay in motor intention and its execution require installation of counter-reactive elements such as motorised braces for the safety of the joint.

2. The detection of intention, which is based on a part of the nerve ensemble (surface electrode to measure EMG) is responsible for movement. While the CNS manages the motor execution in a manner that avoids saturation or stretching of muscle fibres, these fibres change depending on the desired manner of executing the movement (in terms of speed, time, force, etc.). Although this disadvantage can be overcome, the servo-assistance circuits are not operational in cases of amputation or limb paralysis. Thus, given long-term variation in motor commands, reliable detection of motor intention is almost impossible.

We propose another way of detecting motor intention instead of reconstituting it from a partial image of motor execution in the absence of servomotor circuitry (paralysed or amputated limb). We attempted to induce neuro-programming in the motor planning of agonist muscles of a healthy joint. In fact, the actuators of a healthy joint are stable given the normal functionality of the counter-reactive servomotor circuitry. This stability provides highly reproducible SEMG signals from antagonist muscles that support this joint. We only needed to identify a characteristic of these signals, which can be voluntarily controlled during motor action without any major perturbation. Of note, especially in sports and martial arts, motor action can be executed at several antagonist co-activation levels, which can be controlled by long-term training. We decided to take this characteristic as an analog output of the CNS and communicate its motor intention to the myo-electrical system (see Fig. 3).

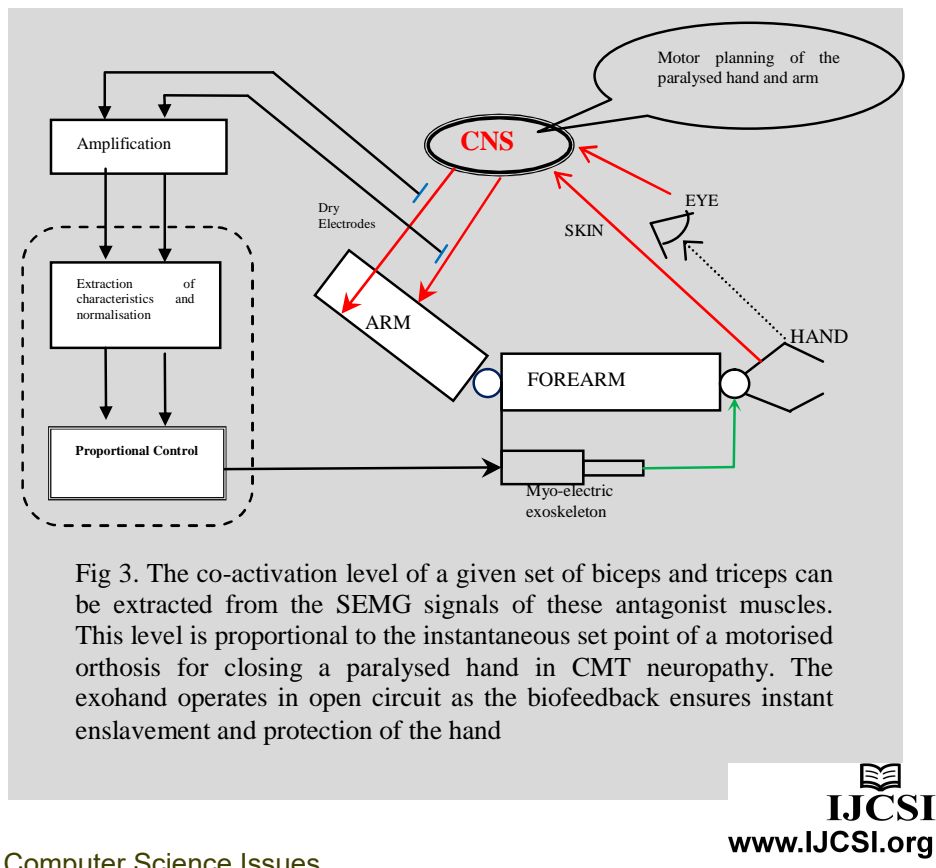


In this method, the CNS regulates the amplitude of the angle of the paralyzed joint and its temporal evolution using circuits against existing visual and sensory counterreactions. This system allows us to have:

1. Real time myo-electric control without classifying the SEMG signals, as it only requires extracting the envelopes of the SEMG signals of antagonist muscles, which are directly related to the level of antagonist co-activation that we demonstrate in the following section.

2. Use of biofeedback allows working in open circuit at the level of the exoskeleton as it enslaves the CNS, which manages the motor action to protect the joint against any possible dislocation.

To prove that such control is possible at the level of antagonist co-activation, we studied the antagonist coactivation of biceps and triceps in a population of different ages and gender.

\section{Materials and Methods}

Right from the start of the phenomenon of motor coordination to executing a movement, for example, to smash a tennis ball, the biceps and triceps increase their forces proportionally to firmly maintain the angle of the elbow that is not directly connected to the closing of the hand. This antagonist activation is more or less voluntarily controlled as it cannot be cancelled. In fact, the CNS automatically generates a minimum holding force to protect the joint from exceeding the limits of the angles; however, one can regulate the maintenance level up to the maximum voluntary force (MVF). The regulation of antagonist co-activation is achieved through gradual training- a phenomenon observed in athletes and musicians who manage to control their motor coordination for the perfect execution of movement with least effort.

Voluntary control of the force of antagonist co-activation is possible with neuro-motor programming so that we can take it as a myo-electric control setup of support equipment for a paralyzed joint. The right choice of muscle antagonists that will support this myo-electric command is essential to facilitate natural movement execution. In our view, the controller muscles must be those that are most sought after by the CNS in the motor planning of the paralysed part of the limb.

To illustrate this possibility; we studied a healthy population of different ages and gender, to determine neuro-motor programming based on biofeedback aimed at improving the regulation of antagonist co-activation of biceps and triceps.
Neuro-motor programming has two objectives:

1. The antagonist muscles should hold the elbow at the same angle, at several levels of co-activation ranging from minimum force to maintaining the maximum voluntary force (MVF).

2. Flexing and elbow extension must be executed while keeping the level of antagonist co-activation constant as displayed by SEMG.

Regulating these two factors allows proportional control of antagonist co-activation without influencing the motor planning of the elbow. The first factor allows control of the static mode and the second factor regulates the dynamic mode of the elbow.

We attempted to develop a simple elbow control model to demonstrate that co-activation of antagonist biceps and triceps are voluntarily controlled at a fixed angle, or during normal flexing and extension without acceleration.

In 1984, Prof. N. Hogan [16] put forth a simple mechanical model (Fig $\mathrm{x}$ ) to demonstrate the relationship between a pair of biceps and triceps, and the angle.

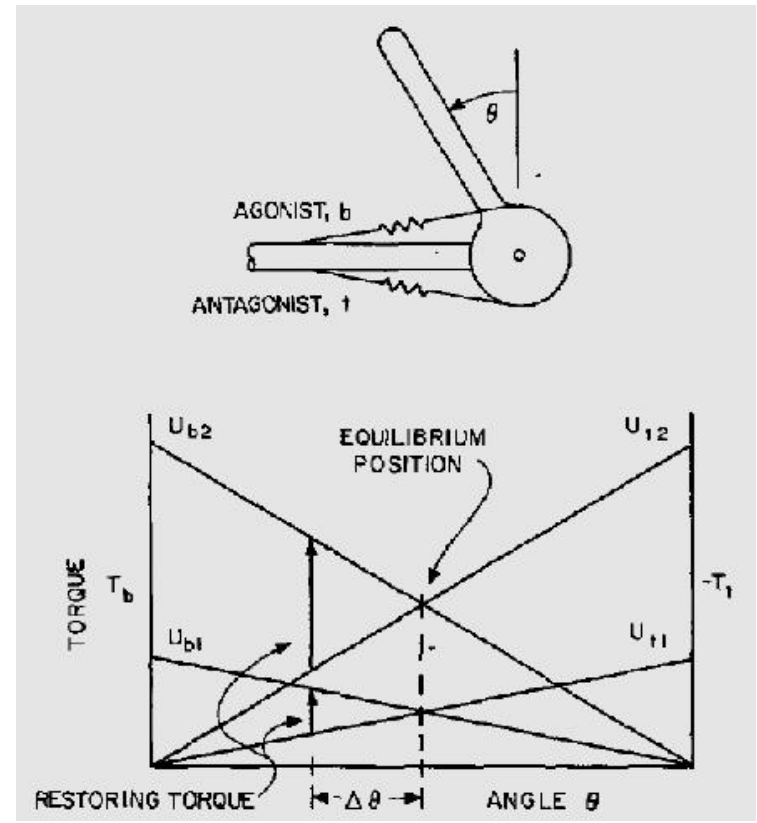

Fig 4 .

According to N. Hogan [16]

$T b=(T-K * \theta) * U b$

$T t=-(T+K * \theta) * U t$ 
Using the same principle and analysing the various explanations for the phenomenon of antagonist coactivation, we propose a hydraulic model that simplifies the relationship between the elbow and the hand (elbow finesse) to facilitate the adoption of the myo-electric exoskeleton by the CNS.

We constructed this model based on the energy efficiency principle of the CNS. In fact, the CNS grants the minimum force required to execute the elbow movement while protecting against any possible elbow dislocations (e.g., strong acceleration, sudden change in the direction of the movement). This CNS force is primarily under voluntary control, and is known as the Holding Force (HF). This force is divided between the antagonist biceps and triceps to animate either flexing and extending, or to maintain a fixed elbow angle. We theoretically and experimentally demonstrate that $\mathrm{HF}$ supports our research findings on proportional control.

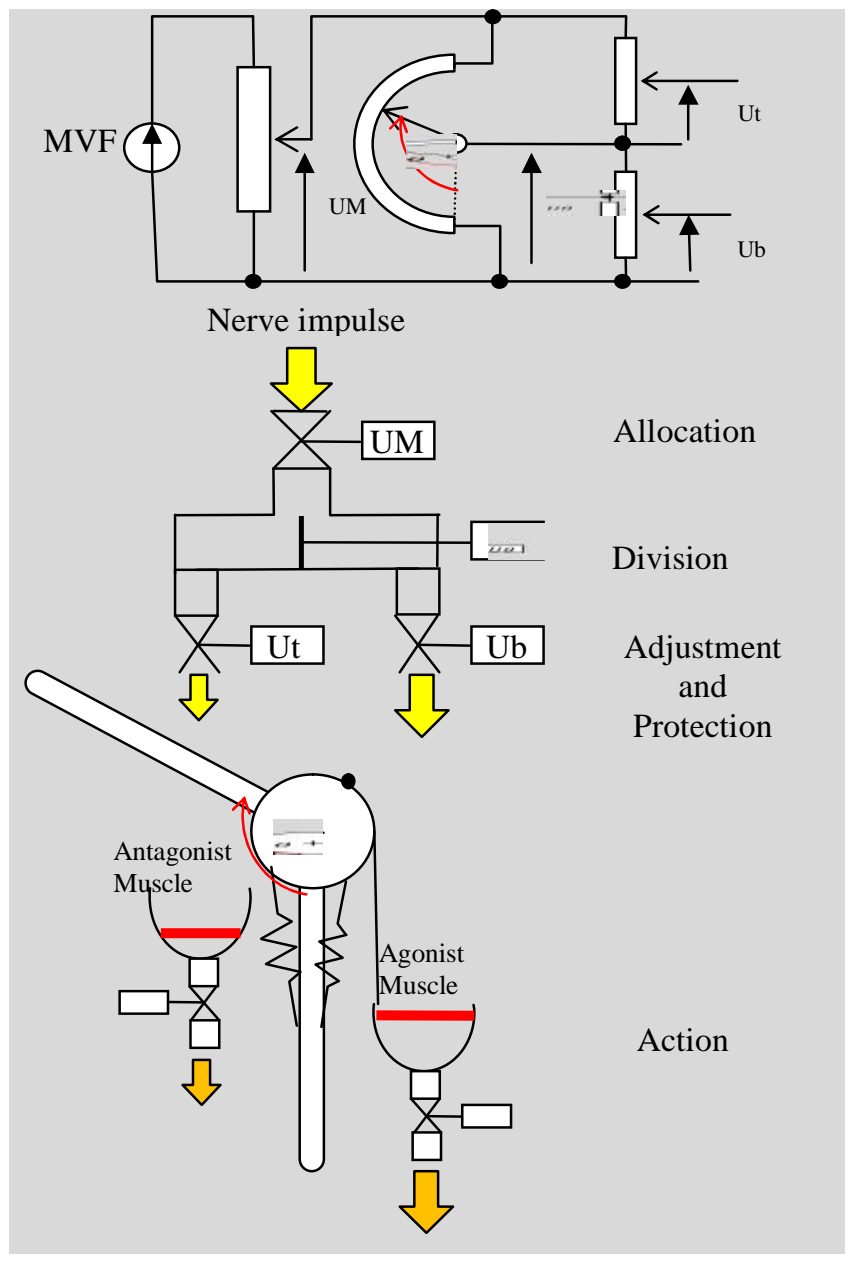

Fig 5 .
The proposed model (Fig 5) consists of four parts:

1. Allocation: This part located in the CNS allows allocating a percentage of MVF for the execution of a given movement. We have symbolised this function by a proportional valve that regulates the flow of nerve impulses. The valve is controlled by a potentiometer powered by an MVF value generator. Hence the relationship:

$$
\begin{gathered}
F M=M V F * U M \\
U M=\frac{F M}{M V F} \\
0 \leq U M \leq 1
\end{gathered}
$$

This unit UM is the subject of our research. We studied the controllable ranges of variation in four positions $\left(0^{\circ}, 45^{\circ}, 90^{\circ}, 140^{\circ}\right)$, and the outstanding stability of the static movement of the elbow (from $0^{\circ}$ to $140^{\circ}$ ).

2. Distribution: Also located in the CNS, this part is responsible for distributing the allocated force between the biceps and triceps. In our model, this role is performed by a distributor that is controlled by a rotating potentiometer, which also symbolises the generation of the inverse function of the command for a chain of high level counterreactions.

The consequent relations are:

$$
\begin{gathered}
F 1=U \theta * F M \\
F 2=(1-U \theta) * F M \\
U \theta=\frac{\theta}{\theta \max } \\
0 \leq \theta \leq \theta \max \\
0 \leq U \theta \leq 1
\end{gathered}
$$

3. Adjustment and Protection: These functions are performed by neuro-motors, which adjust the distribution based on the influence of external forces gravity, mass, etc.,) or the forces induced by acceleration and changes in the direction of voluntary movement, or by reflex. Thus, the final formulae for the command options are:

$$
\begin{aligned}
F b & =F 1 * U b \\
F t & =F 2 * U t \\
0 & \leq U b \leq 1 \\
0 & \leq U t \leq 1
\end{aligned}
$$

The coefficients, Ub and Ut, characterise the low level counter-reaction system (neuro-motor). These coefficients will be introduced halfway to facilitate the increase and decrease operation, as required. For our purposes, we required a valid set of static movements and outstanding standards to retrieve the maximum controllable mobility. For this reason, these coefficients were considered constant and equal to 1 . 
4. Action: This part models the mechanical part of the elbow. N. Hogen's model has two containers/collectors that receive neuro impulses delivered by proportional valves. These containers are equipped with a discharge valve for getting rid of the load accumulated at the end of the action. The springs represent the passive force of the muscle as opposed to the containers, which represent the active force. The capacity to accumulate and discharge the nervous flux (charge) depends on the type of muscular fibres. Thus the containers should be sized proportionally.

The following formulae can be deduced from our model:

$$
\frac{F b}{M V F}=U M * U \theta
$$

$$
\begin{array}{r}
\frac{F t}{M V F}=U M *(1-U \theta) \\
U M=\frac{F b+F t}{M V F} \\
U \theta=\frac{F b}{F b+F t}
\end{array}
$$

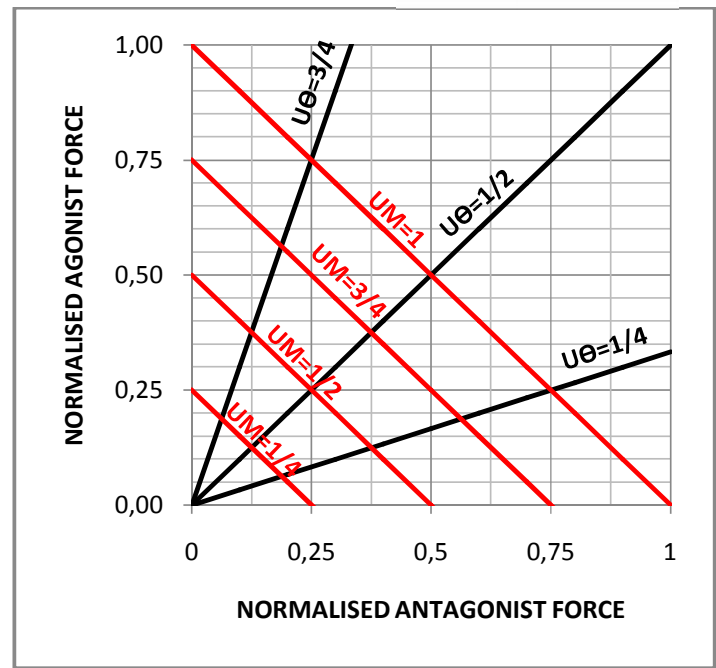

Fig 6:

These studies on the two functions $U M(F b, F t)$ and $U \theta(F b, F t)$ allow us to conclude that:

1. UM varies from 0 tol regardless of

2. $\mathrm{U}$ varies from 0 to 1 regardless of FM

Thus, the voluntary variation of UM regardless of the angle of the elbow, and the control of UM during the static movement of the elbow, prompted us to take an analog set from 0-1 for controlling an automated system.

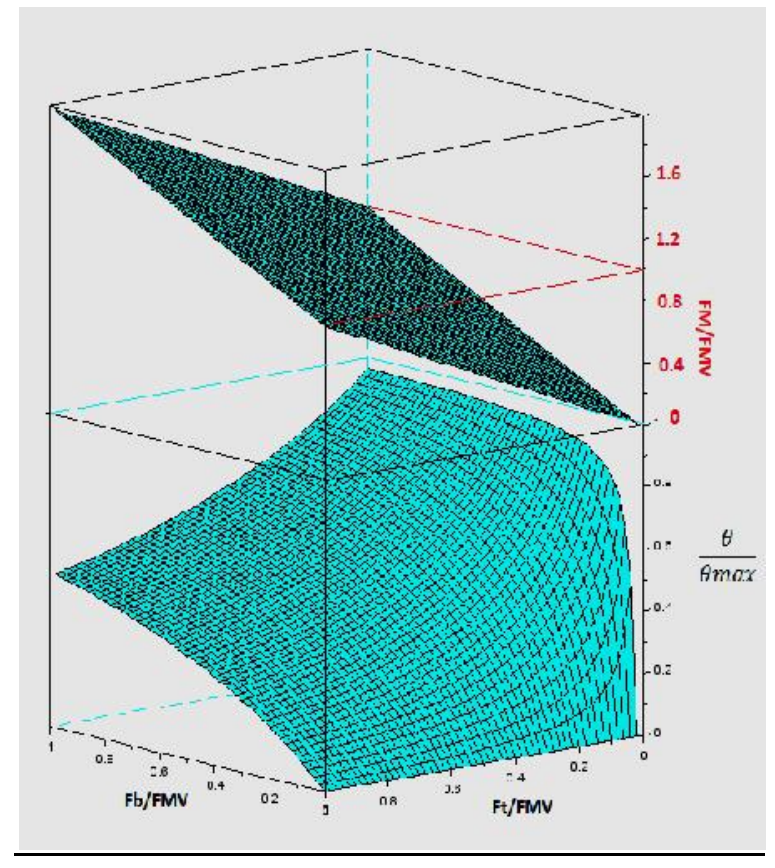

Fig: 7

\subsection{Test Tool}

To prove the reliability of this proportional control, we developed a surface electromyogram that detects SEMG signals from biceps and triceps, and measures the angle of the elbow. The measured values were normalised to the maximum value with the signal processing system. These normalised measured values are displayed in real time.

Thus the system developed consists of: Hardware

1. Sensors:

- Two bipolar dry electrodes

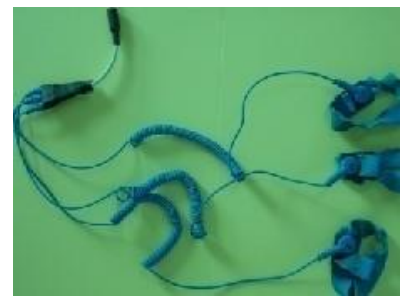

- Two EMG amplifiers

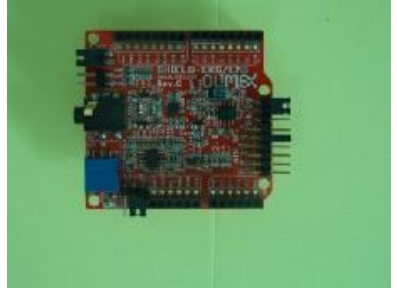


2. Controller:

- Arduino microcontroller

4. Power supply:

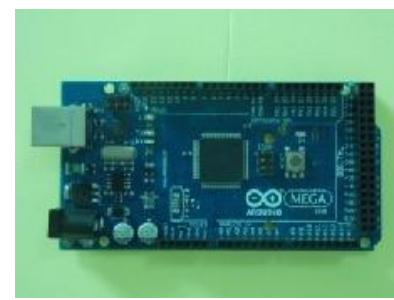

- $\quad$ Spring blade

- Two 6V 1Ah batteries

3.2

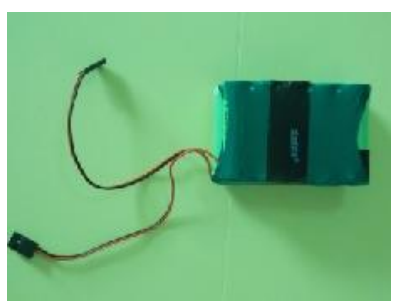

Assembly

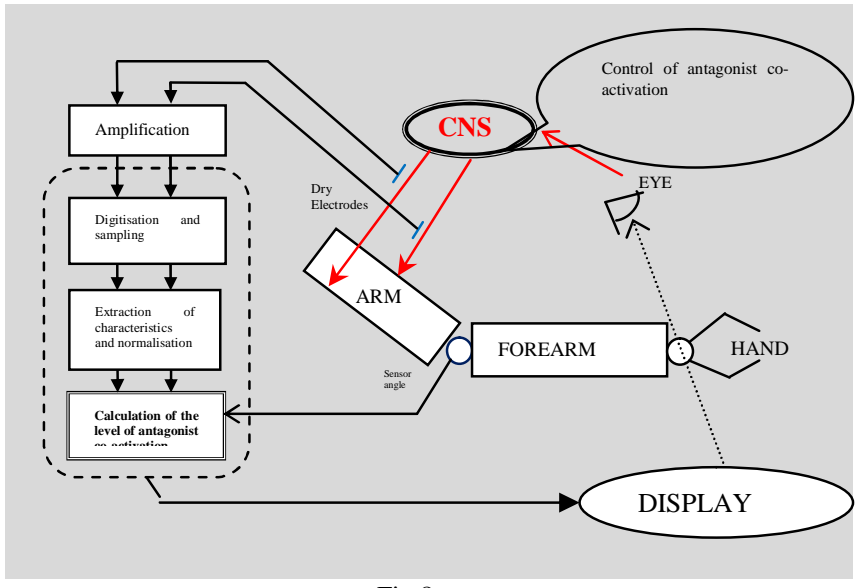

Fig:8

\subsection{Software}

We developed a digital $2 \mathrm{~Hz}$ low pass filter that passes a value representative of the force.

1. $2 \mathrm{~Hz}$ high pass filter

$$
S_{0}[n]=S_{0}[n-1]+\frac{E[n]-S_{0}[n-1]}{N}
$$

$$
\begin{gathered}
N=\frac{T_{f}}{T_{e}} \\
S_{1}[n]=E[n]-S_{0}[n]
\end{gathered}
$$

$\mathrm{E}[\mathrm{n}]$ : Input signal (amplified SEMG)

Tf: Response time of filter $(500 \mathrm{~ms})$

Te: Sampling period (1ms)

$\mathrm{S}_{0}[\mathrm{n}]$ : Continuous component of signal $\mathrm{E}[\mathrm{n}]$

$S_{1}[n]$ : Alternative component

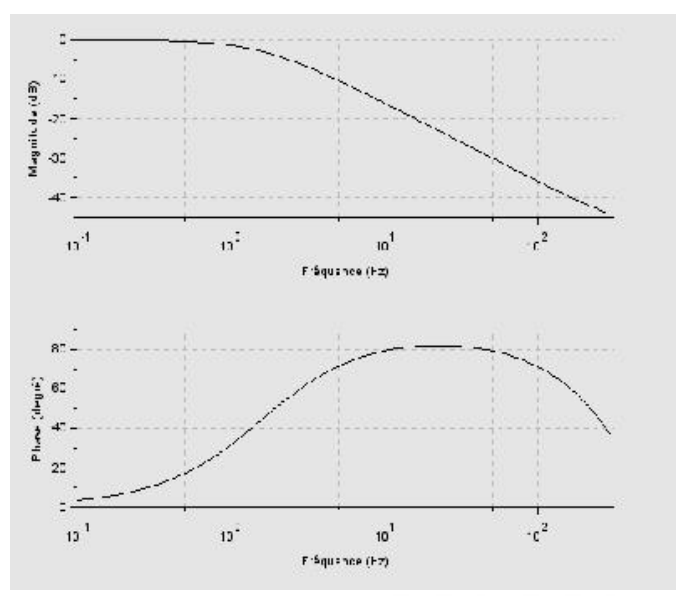

2. Recovery

$$
S_{2}[n]=\left|S_{1}[n]\right|
$$

3. Extraction envelope

$$
\begin{gathered}
\text { si } S_{3}[n-1]<S_{2}[n] \Rightarrow S_{3}[n]=S_{2}[n] \\
\text { si } S_{3}[n-1] \geq S_{2}[n] \Rightarrow S_{3}[n]=S_{3}[n-1]-\mathrm{K}
\end{gathered}
$$

4. $2 \mathrm{~Hz}$ low pass filter

$$
\begin{gathered}
S_{4}[n]=S_{4}[n-1]+\frac{S_{3}[n]-S_{4}[n-1]}{N} \\
N=\frac{T_{f}}{T_{e}}
\end{gathered}
$$

5. Normalisation

During the first 10 seconds, the patient had to develop his/her maximum voluntary agonist and antagonist force, which the software will save as

$$
\begin{gathered}
\text { si } S_{\max }<S_{4}[n] \Rightarrow S_{\max }=S_{4}[n] \\
F_{b}=\frac{S_{4}[n]}{S_{\max }}
\end{gathered}
$$

6. Proportional Control

$$
\begin{gathered}
F M[n]=F_{b}[n]+F_{t}[n] \\
C P[n]=C_{\max } \times(1-F M[n])
\end{gathered}
$$




\subsection{Organisation Chart}

\section{5}

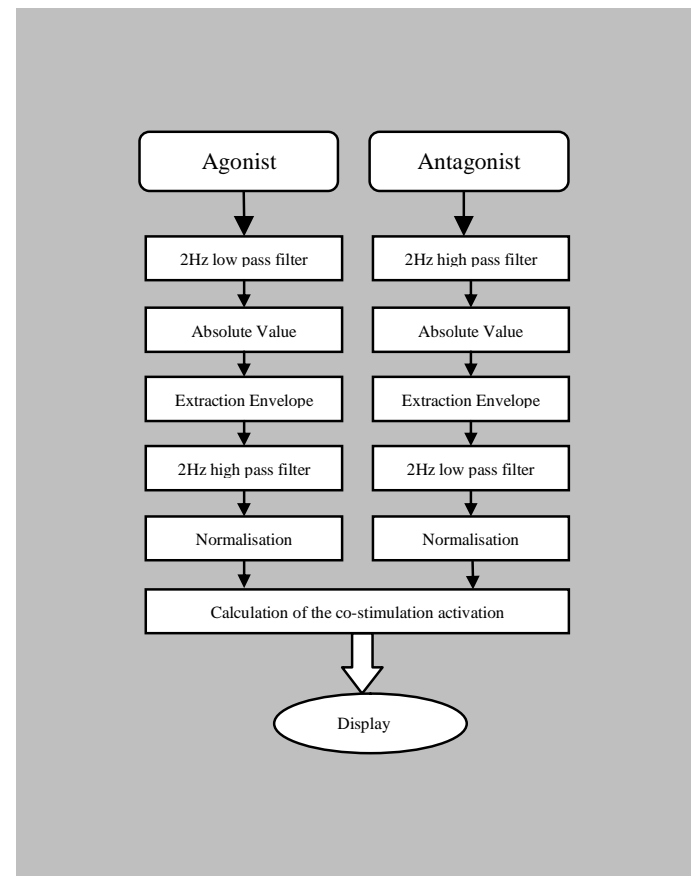

Tests

We studied the possibility of obtaining proportional signal control from SEMG signals of biceps and triceps for which, we performed the following tests on patients:

1. Varying the holding force of the fixed elbow angle $\left(0^{\circ}, 45^{\circ}, 90^{\circ}, 135^{\circ}\right)$ for $10 \mathrm{~s}$.

2. Controlling the holding force during elbow movement (from 0 to $135^{\circ}$ and from $135^{\circ}$ to $0^{\circ}$ ) for $10 \mathrm{~s}$.

\subsection{The test subjects}

In the first instance, we conducted these tests on healthy subjects to validate the method on different age and gender groups. All tests were performed while subjects were seated with an unsupported arm. Real time display of maximum force (MF) and angle values provided biofeedback of the tests.

\section{Results}

The following results were obtained by applying this method to control the opening and closing of the hand using SEMG signals from biceps and triceps:

1. The holding force varied from $10 \%$ to $100 \%$ of the maximum voluntary force
2. Knowing the opening angle $X \sim \mathrm{k}^{*}(\mathrm{~L} 1-\mathrm{L} 0)$ within this range [L0-DL,L0+DL], we obtained linear control of hand opening

3 . In the case of forearm movement, the variation in hand opening was controlled by the choice of the signal $(\mathrm{FM}=\mathrm{F} 1+\mathrm{F} 2)$ and by correctly estimating $\mathrm{DL}$ and $\mathrm{MVF}$, as $\mathrm{dL} 1 / \mathrm{dFM}=\mathrm{DL} / \mathrm{MVF}$

4. In the case of weight lifting, the maintenance force was always controllable, thus maintaining the position of the hand.
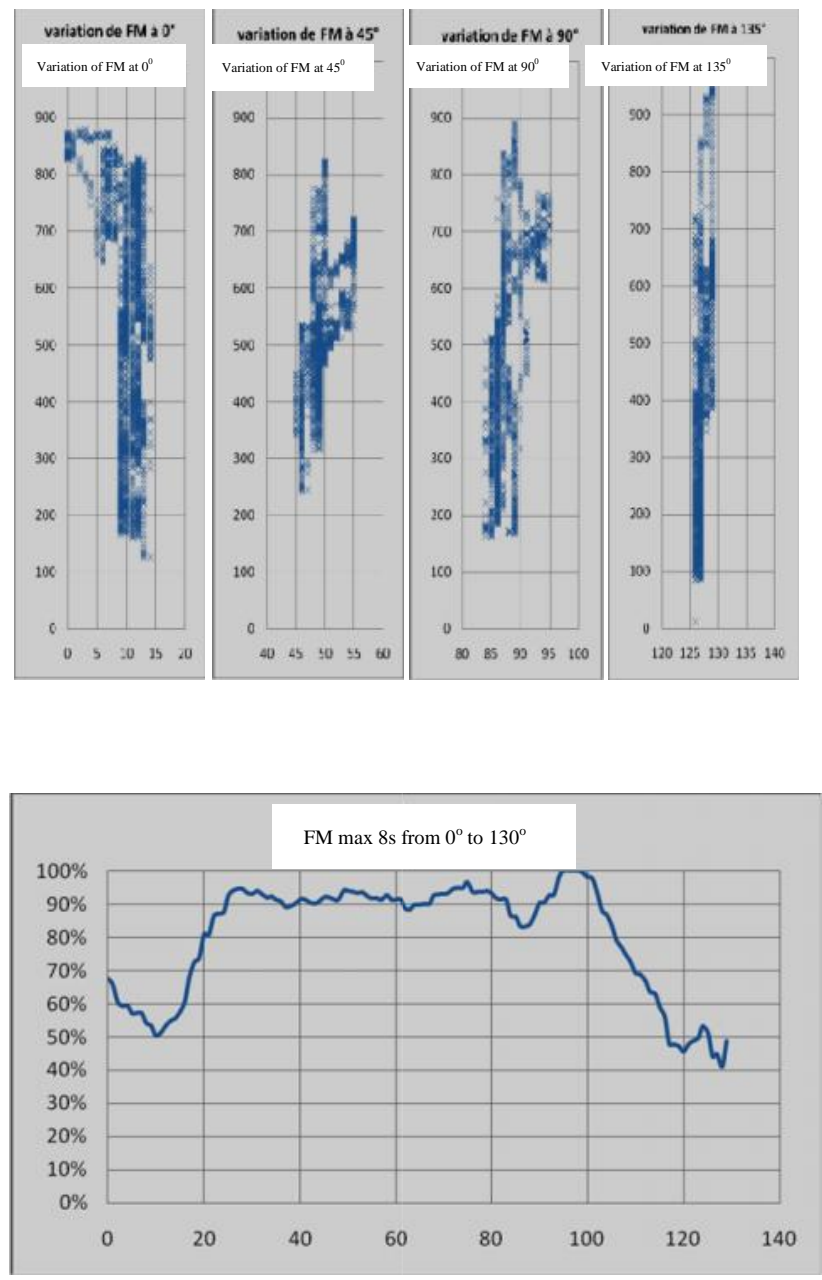
IJCSI International Journal of Computer Science Issues, Volume 14, Issue 1, January 2017 ISSN (Print): 1694-0814 | ISSN (Online): 1694-0784
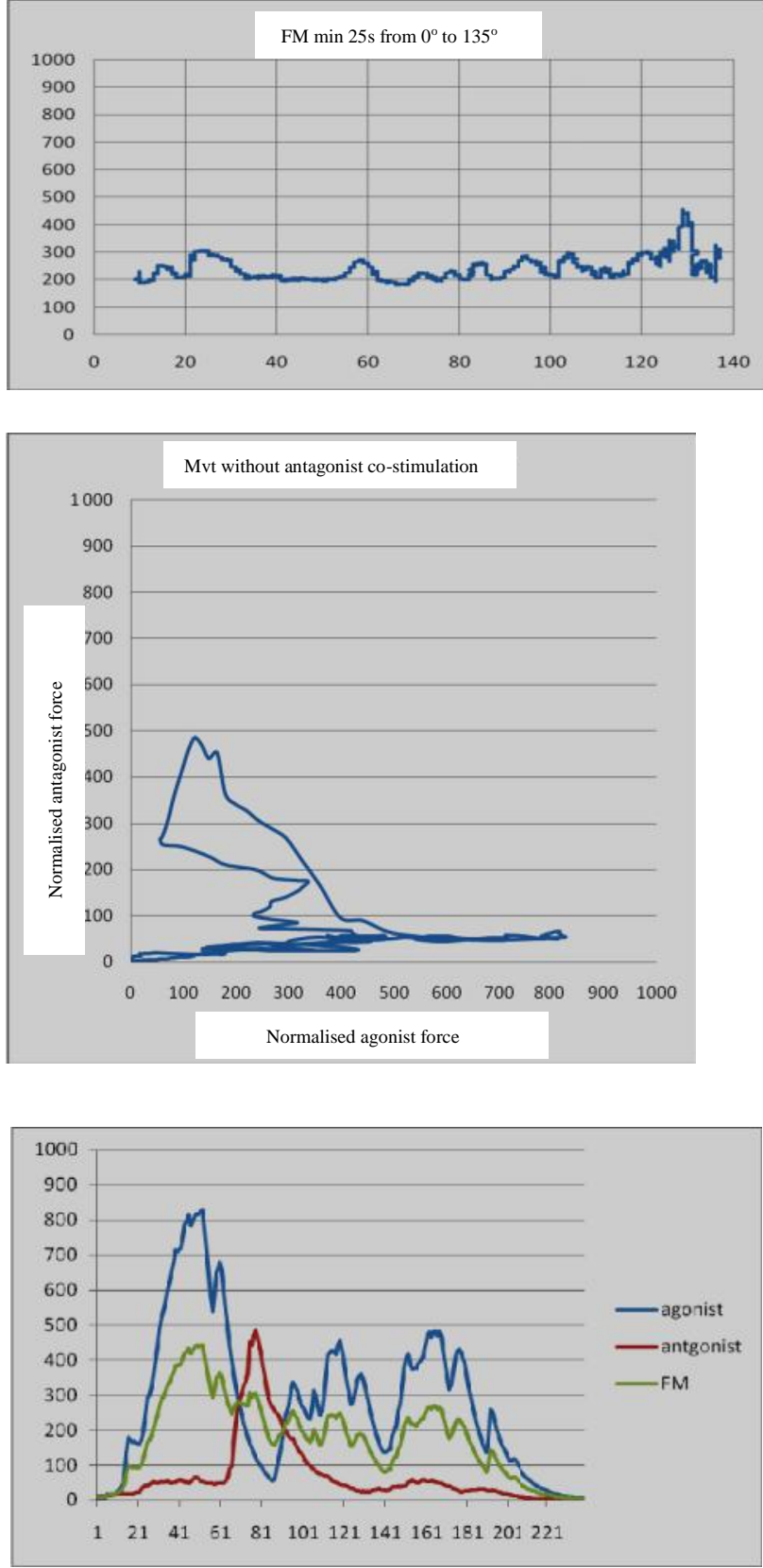
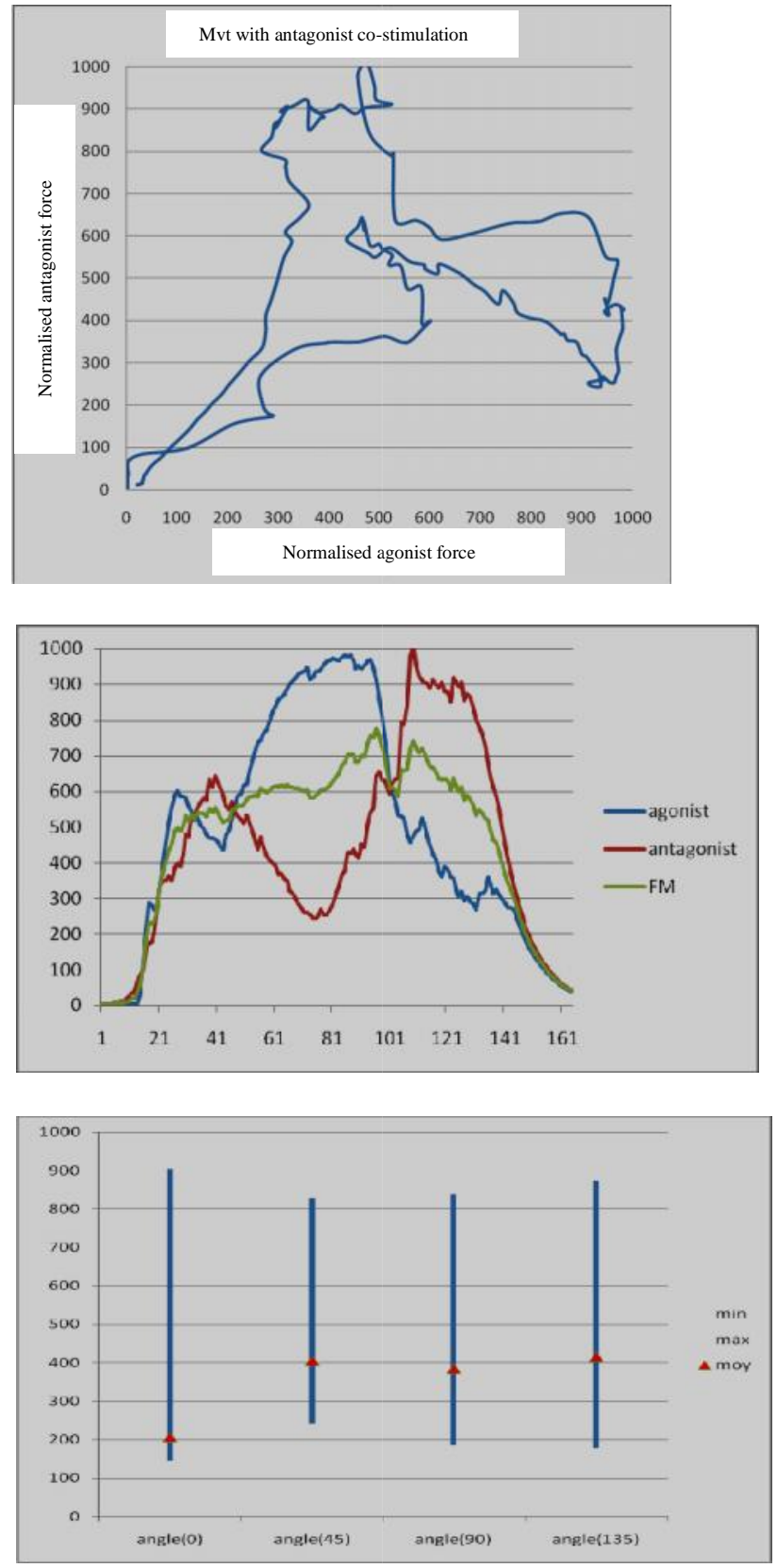


\section{Discussion}

This method has many advantages compared to the other methods described in the first section. First of all this method givesthe possibility of myoeletriccontrol of exoskeleton in cases of peripheral nerve diseases (e.g., CMT). Moreover, it isa simple system that does not requireANN classification software for SEMG signals and finally it makes it possible to avoid taking orders from the central nervous system (CNS).

On the other hand, some disadvantages are also possible mainly the fact that the high holding force can strain the muscles. We may also observe that coordinating simultaneous movement is difficult, but with training, the patient can learn to control several holding forces.

\section{References}

[1] «Avancées dans la maladie de Charcot-MarieTooth $\gg$ juin 2016 - CMT France n.d. http://www.cmtfrance.org/Avancees-dans-la-maladie-de-Charcot-Marie-Toothjuin-2016 (accessed December 16, 2016).

[2] Mhandi E, Feasson, Gautheron, Clamels. Exercice musculaire et maladie de Charcot-Marie-Tooth. - Moteurline n.d. http://www.moteurline.apf.asso.fr/spip.php?article1048 (accessed December 16, 2016).

[3] HUET S. Classification de Signaux Myóelectriques 'etude bibliographique. 2004.

[4] Raez MBI, Hussain MS, Mohd-Yasin F. Techniques of EMG signal analysis: detection, processing, classification and applications. Biol Proced Online 2006;8:11-35. doi:10.1251/bpo115.

[5] Ahmad SA, Ishak AJ, Ali SH. Review of Electromyographic Control Systems Based on Pattern Recognition. In: Osman NAA, Abas WABW, Wahab AKA, Ting H-N, editors. 5th Kuala Lumpur Int. Conf. Biomed. Eng. 2011, Springer Berlin Heidelberg; 2011, p. 556-9.

[6] Mozaffari Foumashi M, Troncossi M, Parenti Castelli V. State-of-the-Art of Hand Exoskeleton Systems 2011. http://amsacta.unibo.it/3198/ (accessed December 16, 2016).

[7] Light CM, Chappell PH, Hudgins B, Engelhart K. Intelligent multifunction myoelectric control of hand prostheses. J Med Eng Technol 2002;26:139-46. doi:10.1080/03091900210142459.

[8] B. Hudgins, P. Parker and R. N. Scott, "A New Strategy for Multifunction Myoelectric Control," IEEE Trans. Biomed. Eng., vol. 40, pp. 82-94, Jan. 1993.

[9] K. Englehart and B. Hudgins, "A Robust, Real-time Control Scheme for Multifunction Myoelectric Control," IEEE Trans. Biomed. Eng., vol. 50, pp. 848-854, Jul. 2003.

[10] L. Hargrove, E. Scheme, K. Englehart and B. Hudgins, "Multiple binary classifications via linear discriminant analysis for improved controllability of a powered prosthesis," IEEE Trans. Neural Syst. Rehabil. Eng., vol. 18, no. 1, pp. 49-57, Jan 2010.

[11] A. Fougner, E. Scheme, A. Chan, K. Englehart and Ø. Stavdahl, "Resolving the Limb Position Effect in Myoelectric Pattern Recognition," IEEE Trans. Neural Syst. Rehabil. Eng., vol. 19 , no. 6 , pp. 644-651, 2011.
[12] Scheme E, Lock B, Hargrove L, Hill W, Kuruganti U, Englehart K. Motion Normalized Proportional Control for Improved Pattern Recognition-Based Myoelectric Control. IEEE Trans Neural Syst Rehabil Eng 2014;22:149-57. doi:10.1109/TNSRE.2013.2247421.

[13] Antfolk C, Cipriani C, Controzzi M, Carrozza MC, Lundborg G, Rosén B, et al. Using EMG for Real-time Prediction of Joint Angles to Control a Prosthetic Hand Equipped with a Sensory Feedback System. J Med Biol Eng 2010;30:399406. doi:10.5405/jmbe.767.

[14] Xiao Z, Menon C, Khokhar Z. Surface EMG Pattern Recognition for Real-Time Control of a Wrist Exoske BioMedical Engineering OnLine 2010, 9:41

http://www.biomedical-engineeringonline.com/content/9/1/41leton 2010 .

[15] A. Simon, K. Stern and L. Hargrove, "A Comparison of Proportional Control Methods for Pattern Recognition Control," in Proceedings of EMBS Conference, Boston, 2011.

[16] Hogan N. Adaptive control of mechanical impedance by coactivation of antagonist muscles. IEEE Trans Autom Control 1984;29:681-90. doi:10.1109/TAC.1984.1103644.

[17] Remaud A, Guével A, Cornu C. Coactivation et inhibition musculaire : influences sur la régulation du couple de force développé et les adaptations induites par un entraînement en force. Neurophysiol Clin Neurophysiol 2007;37:1-14. doi:10.1016/j.neucli.2007.01.002.

[18] Cao H. Modelisation and experimental evaluation of the relationship between surface EMG signal and muscle force. Theses. Université de Technologie de Compiègne, 2010.

[19] Eskiizmirliler S, Papaxanthis C, Pozzo T, Darlot C. Un modèle de contrôle cérébelleux des mouvements de pointages de l'avant bras humain. ResearchGate, 2006.

[20] PHYSIOLOGIE MUSCULAIRE J-M Mienville, UNS n.d. http://docplayer.fr/12122093-Physiologie-musculaire-j-mmienville-uns.html (accessed December 16, 2016).

Abdelali LAAMARTI is engineer of state in micro-electronics and automatic from Polytechnic Montpellier French with 23 years experience in industry. PHD student in neuroscience since 2012

Pr Abdelhakim Lakhdar member of Laboratory research on nervous system, neurosensorial diseases and handicap.

Pr Omar Battas member of Laboratory research on Neurosciences and mental disease.

Pr Imane Kermadi professor in neuroscience.

Pr Mohammed Abdoh Rafai member of Laboratory research on nervous system, neurosensorial diseases and handicap. 